\title{
Application of Information Technology in Operation and Maintenance of Overhead Transmission Lines
}

\author{
Li Xiaoguang ${ }^{1, a}$, Wang Xiaofu ${ }^{1}$, Liang Naifeng ${ }^{1}$,Deng Yuanjing ${ }^{2, b^{*}}$ \\ and Xia Kaiquan ${ }^{2}$ \\ ${ }^{1}$ State Grid Xinjiang Electrical Power Company \\ 830002, Urumqi, Xinjiang, China \\ ${ }^{2}$ China Electrical Power Research Institute \\ 100055,Beijing, China \\ a75680875@qq.com, bdeng_yuanjing@163.com
}

\begin{abstract}
Keywords: Condition based maintenance, Operation and maintenance, Online-monitoring, Information, Big data

Abstract. Operation and maintenance management work of overhead transmission lines is related directly to safety and reliability of the power grid. As overhead transmission lines are a significant composition part of smart gird, improvement of the operation and maintenance of management is an important measure to support smart grid. This discussion of operation and maintenance of overhead transmission lines are carried out combined with the demand of the current situation and new information technologies. It is appointed that innovation and advancement of the operation and maintenance mode can be pushed forwarded fully by means of on-line monitoring, live detection technology and information technology according to status and assessment of the transmission lines. It is forwarded that deepening application of the information technology is the key to improve efficiency and quality of operation and maintenance of the line and improve management level of the with the big data technology.
\end{abstract}

\section{Introduction}

The overhead power transmission line is an important infrastructure facility in the power industry, which undertakes important task for power transmission. With rapid development of the grid construction, scale of the grid is enlarged gradually, coverage scope of the power transmission line is brand, and most are operating at urban and open area, it is often affected by severe weather, geographical conditions which endanger safe and reliable operation of the grid. As continuous development of the smart grid, the on-line monitoring system of the transmission lines is extensively applied. It actually improves management level of estate in the power corporation, and enhances operation reliability of the grid. And also improve the master and control capability of the dispatch, operation and inspection staff. Abnormal conditions which threaten safety of the line are found out in time, which provides support for risk exclusion and condition based maintenance. At present, it is an important method to improve operation and maintenance efficiency and quality, improve management level of the transmission lines by means of innovation and combination of the operation and maintenance mode and the new information technology.

\section{Current situation and existing issues on operation and maintenance management of transmission line}

It is a key issue to avoid accidental power-off time at maximum level, reduce maintenance cost and prolong service life in operation and maintenance management of transmission lines in the power system. Operation and maintenance management of the overhead transmission lines mainly consists of inspection, status detection and condition based maintenance. In which, condition assessment is an important stage. On-line monitoring, live detection, inspection and other means are main method for collection the relevant information of transmission line. 
Principle of condition based maintenance. Condition based maintenance (CBM) means to compile maintenance plan according to condition assessment. Implementation of CBM is realized through inspection, detection, test, or on-line monitoring, live detection etc means. CBM has been completely applied in the operation and maintenance management and it solves "excessive maintenance" existing and low operation efficiency and economic benefit etc problems caused by period maintenance. With widely application of the sensor technology, digital information processing technology and expert system etc intelligent information technology in the on-line monitoring, live detection and status evaluation, CBM is popularized and improved.

Inspection. According to regulations of DL/T741-2010 Operation regulations of overhead power transmission line (abbreviated as Operation regulations), inspection is to check the line corridor, ambient environment, crossing along the line and construction conditions etc so as to find out and master dynamic change of the line corridor environment in time.

At present, inspection is also assisted by the helicopter, the unmanned aerial vehicle. In order to improve efficiency and reduce labor strength of the patrol inspection person, State Grid Corporation has popularized testing of coordination inspection mode of the helicopter, the unmanned aerial vehicle and manned inspection, and carried out three-dimensional inspection, in particular to inspection of the $500 \mathrm{kV}$ line.

Inspections of the helicopter and the unmanned aerial vehicle mostly apply the far infrared thermal imaging instrument and the visible light camera etc advanced equipments and they are not limited by geographic terrain, some hidden line fault which is difficult to find out during manned inspection, and computer data analysis can be carried out through the configured system.

On-line monitoring system and live detection. The on-line monitoring technology is a process to obtain criteria through monitoring of the line in service and the corridor environment by means of the sensor installed on the transmission lines. Data collected by the on-line monitoring system deployed by State Grid Corporation include three large categories and 10 sub-type data of the transmission line, i.e., image/vide monitoring, micrometeorology, icing thickness monitoring, inclination of tower monitoring, temperature of the conductor, sag of the conductor, breeze vibration of the conductor, galloping of the conductor, filth degree of the insulator and wind deviation of the conductor etc. At current phase, data of the online monitoring system are mostly applied to support operation and assistant of inspection. Status monitoring data are mainly applied in status warning, early warning of disaster and condition assessment of the power transmission line.

At same time, the intelligent robot is currently applied which inspection route can be set or operated by manual, the installed detection system is applied to transmit detection data back to the backstage. Besides reliability issue of the device proper body in the on-line monitoring system, stability of the communication system is also an important issue to restraint data application.

Live detection means process to obtain criteria with contact or non-contact way during operation of the electrical devices. It is not necessary to power off during live detection thus will not affect reliability of power supply. And the far infrared detection technology, the ultraviolet discharging detection technology and the lightning arrestor live detection etc are applied to transmission lines.

\section{Information and big data technology during operation and maintenance of power transmission line}

The smart grid is considered as one of the most important application field of the big data. With development of construction of the smart grid in State Grid Corporation, data quantity which shall be processed and analyzed are increased greatly because the information system is deeply applied in power system, such as online monitoring system of transmission and transformation system and electric information acquisition system. Meanwhile, new innovation on analysis, simulation, forecast and planning, operation, monitoring and control etc aspects on basis of the obtained data are required, which will establish theoretical and technical foundation to realize development target of the smart grid.

With rapidly development of information technology popularization, the intelligent KWh meter, the on-line monitoring system of the power device, the movable detection devices, the measurement 
and control integration system and a large quantity of information management systems serving every specialty are gradually established and applied, scale and kind of the data are increased quickly.

Data generated during operation and maintenance process consists of data generated during inspection, on-line monitoring and live detection, elimination process. These data are managed by different units/departments, which are characterized with scattered displacement and distributed management. These data are not independent and are correlated and influenced each other, and there are complicate relationship. Such as operation conditions of the power transmission line are affected by climate conditions and power consumption conditions, vice versa they will affect reliability of power supply, and then affect power consumption conditions.

Core value of big data is analysis and data mining. These include date correlation analysis and clustering analysis etc technologies. Because data (including on-line monitoring, live detection, preventive test data etc) volume of the equipment are great, types are variety, the big data technology can be imported to abnormal detection of the equipment. The big data analysis technology provides new-brand solution method and idea for abnormality detection accuracy of the equipments through finding out correlation relationship between information.

And the basis of data processing is the stable and reliable communication system. For example, in online monitoring system of transmission lines the communication system prefers the OPGW and $3 \mathrm{G}$ or Wi-Fi hybrid system.

\section{Application prospect of information technology in operation and maintenance of power transmission line}

Further requirement on operation and maintenance of the power transmission line requests to carry out evaluation quickly, i.e., self-evaluation, which has quick response capability and quickly acts on re-closing or switching off the fault section according to actual accident status, and start the line of the standby section. And with certain defence and warning capability, defence capability means capability of the grid against nature disaster and destruction of external force. Such as: withstand rain, snow and fog and other severe weather, flood, bird and artificial destruction and theft of the power transmission tower etc. And with high operation and maintenance efficiency is obtained, the advanced monitoring and control technology is imported. Realize integration of monitoring, control, patrol inspection, maintenance and inspection etc, and a closed loop operation mode is formed. It is necessary to apply the big data, cloud calculation, the expert system etc intelligent technology and the high speed and stable communication system.

The smart power transmission line is an integration of the physical network and the information network. Therefore the uniform communication and information platform of the power equipment shall be established in the smart gird information system frame, and realize information integration and interaction between transmission lines and the application system in other stage and loop. The more complete grid view is formed through information integration between the line and the substation, which can provide better technical fundamental guarantee for decision and analysis of the intelligent grid. It has significant theoretical meaning and application value.

The helicopter, the unmanned aerial vehicle and manned patrol inspection are applied to assist patrol inspection and the laser radar measurement system on the helicopter (Light Detection And Ranging, LIDAR) is taken advantage of for inspections, this system is characterized with rapidity, non-contact, penetration, whole weather, high precision and high efficiency etc advantages, the three dimension data information with high accuracy in the power transmission line can be obtained, digitalization and visualization of the documents of the power transmission network line can be realized through the wholly digital, intelligent data processing flow in combination with the on-line monitoring system of the power transmission line. It includes re-construction of the three dimension visualization and digitalization power transmission network, the power transmission line corridor, analysis of the spatial distance, automatic safety distance detection of the planetary and the house etc dangerous location and objects etc functions. 


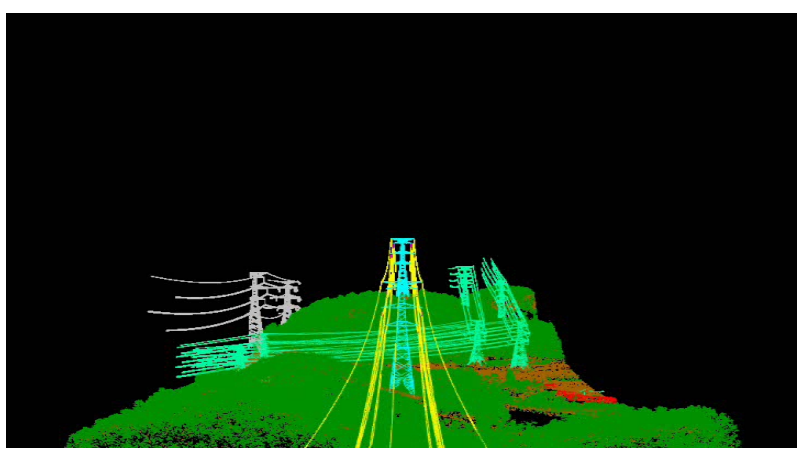

Fig.1 Three dimension re-construction of power transmission line corridor with LIDAR system

The multiple source data are applied to develop status evaluation of the transmission lines. Establish the cloud calculation platform, arrange the power transmission line model and the expert evaluation system, information generated during inspection process, initial design value of the line and the account information can realized intelligent warning, prevention and emergency management on safety of the grid through the expert evaluation system and collaborative mechanism, and operation and maintenance strategy and suggestion are forwarded.

The whole view operation and status information of the power transmission line through with the advanced inspection means and intelligent monitoring method through the high efficiency, reliable and standardized information acquisition technology and communication way, and carry out analysis, evaluation, diagnosis and control of status of the line on such basis, which can realize data share and mutual-action with the intelligent dispatch system, the production management system, the operation and maintenance system, the relevant design companies etc.

\section{Summary}

The general conditions of operation and maintenance management of the transmission lines are summarized and the current existing problems are proposed in this paper. It proposed that information technology and big data technology during operation and maintenance of the power transmission line are required, and points out application prospect of the information technology in transmission lines. It realizes digitalization and visualization of the documents and material of the transmission lines with the actual measured operation and maintenance data, the on-line monitoring data, the live detection data, the inspection data and the test data etc, will contribute to further research of date integration and data mining technology with the big data theory. Fully take advantage of fault theory, status monitoring, and evaluation diagnosis with information technologies will realize intelligent decision and control which will improve the operation and maintenance management level of the power transmission line.

\section{References}

[1] XIE Zhiwu. Study of Online Monitoring Technology for High Voltage Transmission Lines[J]. Chinese Electrical Education, pp. 180-181, April 2013.

[2] SHAO Yunlin, CAO Jinen and SHANG Dawei. The Helicopter inspection of the EHV transmission lines in North China[J]. China Electrical Power, pp. 35-38, 2003, 36(7) .

[3] SHU Yinbiao and HU Yi. The Operation and Maintenance and the Live Working for the EHV AC Transmission Lines[J]. High Voltage Engineering,pp.1-5,2008,33(6).

[4] Deng Yuanjing, Wang Congmin,Xia kaiqua,et al. Technology And Application of Overhead Transmission Line Corridor Inspection[J], Zhejiang Electric Power ,2014:9:28-31.

[5] YU Deming, SHEN Jian, WANG Jun, et al. The Comparation and Analysis of the Effects for Helicopter and Artificial [J]. China Electrical Power, pp. 25-28, 2008, 41(11). 
[6] CHENG Guokai and WANG Congmin. Discussion On the Construction of the State Inspection System of Transmission Lines[J]. Electrical Power in Zhejiang, September 2013, pp. 11-13.

[7] YI Lei and LU Guiwen. The Application of the Airborne Laser Radar In Circuit Design[J]. The science and technology innovation herald, pp. 65-66, 2013, 17.

[8]Deng Yuanjing, Xia kaiquan, Ren Xichun, Zhang Hao. Technology And Application of Overhead Transmission Line Corridor Inspection, Mechatronics Electronic Industrial and Control Engineering, Atlantis Press,2014,pp:1117-1120.

[9]WEN Zhiqiang, SHU Naiqiu, PENG Hui et al. Difference Operation and Maintenance Scheduling Optimization of Transmission Lines [J]. Power Systems Technology ,2015,4(39):1101-1107

[10]Peng Yakai, Cai Liuji, Liao Yuan. Study on communication and positioning technology of intelligent operation and maintenance system in power transmission line[J]. Electro technology, 2011,9:13-15 